Original article

\title{
Insulin-signaling abnormalities in drug-naïve first-episode schizophrenia: Transduction protein analyses in extracellular vesicles of putative neuronal origin
}

\author{
Dimitrios Kapogiannis $^{\mathrm{a}, * *}$, Henrik Dobrowolny ${ }^{\mathrm{b}, \mathrm{c}}$, Joyce Tran ${ }^{\mathrm{a}}$, Maja Mustapic ${ }^{\mathrm{a}}$, \\ Thomas Frodl $^{\mathrm{b}, \mathrm{d}, \mathrm{e}}$, Gabriela Meyer-Lotz ${ }^{\mathrm{b}, \mathrm{c}}$, Kolja Schiltz ${ }^{\mathrm{b}, \mathrm{f}}$, Denny Schanze ${ }^{\mathrm{g}}$, \\ Marcella Rietschel $^{\mathrm{h}}$, Hans-Gert Bernstein ${ }^{\mathrm{b}, \mathrm{c}}$, Johann Steiner ${ }^{\mathrm{b}, \mathrm{c}, \mathrm{d}, *}$ \\ a Laboratory of Neurosciences, National Institute on Aging / National Institutes of Health (NIA/NIH), Baltimore, MD, USA \\ ${ }^{\mathrm{b}}$ Department of Psychiatry and Psychotherapy, Otto-von-Guericke-University Magdeburg, Magdeburg, Germany \\ ${ }^{\mathrm{c}}$ Laboratory of Translational Psychiatry, Otto-von-Guericke-University Magdeburg, Magdeburg, Germany \\ ${ }^{\mathrm{d}}$ Center for Behavioral Brain Sciences (CBBS), Magdeburg, Germany \\ e German Center for Neurodegenerative Diseases (DZNE), Magdeburg, Germany \\ ${ }^{\mathrm{f}}$ Department of Forensic Psychiatry, Ludwig-Maximilians-University Munich, Munich, Germany \\ ${ }^{\mathrm{g}}$ Institute for Human Genetics, Otto-von-Guericke-University Magdeburg, Magdeburg, Germany \\ ${ }^{\mathbf{h}}$ Department of Genetic Epidemiology in Psychiatry, Central Institute of Mental Health, Medical Faculty Mannheim, Heidelberg University, Mannheim, \\ Germany
}

\section{A R T I C L E I N F O}

\section{Article history:}

Received 24 April 2019

Received in revised form 5 August 2019

Accepted 26 August 2019

Available online 4 October 2019

\section{Keywords:}

Schizophrenia

Psychosis

Molecular biology

Exosomes

Insulin

Serine-threonine kinases

AKT

GSK3 $\beta$

mTOR

p70S6K

IRS-1

\begin{abstract}
A B S T R A C T
Background: Metabolic syndrome and impaired insulin sensitivity may occur as side effects of atypical antipsychotic drugs. However, studies of peripheral insulin resistance using the homeostatic model assessment of insulin resistance (HOMA-IR) or oral glucose tolerance tests (OGTT) suggest that abnormal glucose metabolism is already present in drug-naive first-episode schizophrenia (DNFES). We hypothesized impairments of neuronal insulin signaling in DNFES.

Methods: To gain insight into neuronal insulin-signaling in vivo, we analyzed peripheral blood extracellular vesicles enriched for neuronal origin (nEVs). Phosphorylated insulin signal transduction serine-threonine kinases pS312-IRS-1, pY-IRS-1, pS473-AKT, pS9-GSK3 3, pS2448-mTOR, pT389-p70S6K and respective total protein levels were determined in plasma nEVs from 48 DNFES patients and healthy matched controls after overnight fasting.

Results: Upstream pS312-IRS-1 was reduced at trend level $(\mathrm{p}=0.071$; this condition may amplify IRS-1 signaling). Exploratory omnibus analysis of downstream serine-threonine kinases (AKT, GSK3 $\beta$, mTOR, p70S6K) revealed lower phosphorylated/total protein ratios in DNFES vs. controls $(p=0.013)$, confirming decreased pathway activation. Post-hoc-tests indicated in particular a reduced phosphorylation ratio of mTOR $(p=0.027)$. Phosphorylation ratios of p70S6K $(p=0.029), \operatorname{GSK} 3 \beta(p=0.039)$, and at trend level AKT $(p=0.061)$, showed diagnosis-dependent statistical interactions with insulin blood levels. The phosphorylation ratio of AKT correlated inversely with PANSS-G and PANSS-total scores, and other ratios showed similar trends. Conclusion: These findings support the hypothesis of neuronal insulin resistance in DNFES, small sample sizes notwithstanding. The counterintuitive trend towards reduced pS312-IRS-1 in DNFES may result from adaptive feedback mechanisms. The observed changes in insulin signaling could be clinically meaningful as suggested by their association with higher PANSS scores.
\end{abstract}

(c) 2019 Elsevier Masson SAS. All rights reserved.

\footnotetext{
* Corresponding author at: Department of Psychiatry and Psychotherapy, University of Magdeburg, Leipziger Str. 44, D-39120 Magdeburg, Germany.

** Corresponding author at: Laboratory of Neurosciences, Intramural Research Program, National Institute on Aging, NIH, 251 Bayview Blvd, 8C228, Baltimore, MD 21224, USA.

E-mail addresses: kapogiannisd@mail.nih.gov (D. Kapogiannis), johann.steiner@med.ovgu.de (J. Steiner).
}

\section{Introduction}

Post-mortem studies have observed reduced expression of insulin receptors and decreased phosphorylation of the downstream signaling proteins AKT, GSK3 $\beta$ and mTOR in frontal cortices from patients with schizophrenia [1-3]. These findings were interpreted as indications of impaired cerebral insulin sensitivity. 
Wijtenburg et al. [23] suggested that brain insulin resistance plays a role in learning and memory dysfunction in schizophrenia. The latter study analyzed differences in brain glucose using magnetic resonance spectroscopy (MRS) and biomarkers of neuronal insulin resistance in blood extracellular vesicle (EVs) enriched for neuronal origin (nEVs) from chronic schizophrenia patients and controls. EVs are nanoparticles comprised of a lipid bilayer, containing RNA and protein cargo. nEVs cargo also contains numerous intercellular signaling molecules and can cross the blood-brain-barrier in both directions $[5,6]$.

Notably, chronic disease and long-term medication use were potential confounders of the aforementioned studies. However, studies of peripheral insulin resistance using the homeostatic model assessment of insulin resistance (HOMA-IR) or oral glucose tolerance tests (OGTT) suggest abnormal glucose metabolism may also be present in drug-naive first-episode schizophrenia (DNFES) patients, independently from stress, smoking or obesity [7-11]. Moreover, impaired OGTT has been observed in unaffected siblings [12]. Therefore, we hypothesized that impaired cerebral insulin signaling occurs in DNFES.

To gain insight into neuronal insulin-signaling in vivo, we analyzed peripheral blood nEVs which have been considered as a "message in a bottle" from neurons [13], by a well-established protocol used in multiple previous studies [14-23] and presented in detail in Mustapic et al. [5].

Our primary outcome nEV biomarker was the upstream insulin signal transduction protein pS312-IRS-1 (Fig. 1a), an established marker of neuronal insulin signaling that has shown diagnostic potential for Alzheimer's disease (characterized by insulin resistance) $[18,19]$, association with gray matter volume [20], cognition $[19,23]$, and dynamic response to insulin signaling-modifying interventions, such as diet, intranasal insulin and exenatide [14,21,24]. Generally, serine phosphotypes stimulate uncoupling of IRS-1 leading to its degradation [25,26]. In an explanatory fashion, we analyzed the functional counterpart of pSer312-IRS1, pY-IRS-1, which generally promotes insulin-stimulated responses [27] and downstream serine-threonine kinases (AKT, GSK3 $\beta$, mTOR, p70S6K; Fig. 1a) to examine the state of the entire insulin signaling cascade, as previously done [14,21]. We hypothesized a lower ratio of phoshorylated to total levels for these proteins in patients vs. controls, indicating reduced signaling activity as previously suggested by postmortem studies (see above). Moreover, we aimed to test if blood insulin measures and the severity of clinical symptoms showed a statistical interaction with the phosphorylation ratios of the tested insulin signaling proteins in DNFES. Finally, we aimed to explore a possible genetic predisposition of the DNFES cohort for insulin signaling anomalies, e.g. whether genetic variants located in genes coding for the tested signal transduction proteins are present with varying probability in patients and controls.

\section{Methods}

\subsection{Samples}

We studied plasma samples from our previous HOMA-IR-based study [9]. These were collected from February 2008 to March 2010 from all available sequentially admitted acutely ill DNFES inpatients ( $n=24)$. Controls $(n=24$; healthy blood donors and hospital staff and their relatives) came from the same collection period (Table 1). Procedures were IRB approved and written informed consent was obtained. nEV isolation and assays were performed by National Institute on Aging investigators blinded to group.

Psychopathology was assessed using the Positive and Negative Syndrome Scale (PANSS). Psychosis resulting from other medical conditions and substance-induced psychosis was excluded by a thorough medical history, physical examination, routine blood analysis, and screening for illegal drugs [28]. The same examinations were carried out for the controls. Controls were cleared for personal or family history of psychiatric and neurological disorders using the Mini-International Neuropsychiatric Interview [29]. Exclusion criteria consisted of the presence of immune diseases, immunomodulating treatment, cancer, chronic terminal disease, cardiovascular disorders, manifested diabetes mellitus or severe trauma [28].

Blood samples were obtained from fasting subjects around 8:00 a.m. and collected into BD Vacutainer ${ }^{\mathrm{TM}}$ tubes (Becton Dickinson; Heidelberg, Germany), as previously described. Plasma tubes were centrifuged immediately at $1000 \mathrm{~g}$ for $10 \mathrm{~min}$; supernatant aliquots were stored at $-80^{\circ} \mathrm{C}$.

\section{2. $n E V$ isolation and enrichment}

As described [5], nEVs were isolated by a two-step methodology including particle precipitation using Exoquick ${ }^{\mathrm{TM}}$ (System Biosciences) to acquire a pellet of total EVs, followed by enrichment for L1 neural cell adhesion molecule (L1CAM) expression using immunoprecipitation with biotinylated antibodies (CD171, clone 5G3) and Pierce $^{\mathrm{TM}}$ Streptavidin Plus UltraLink ${ }^{\mathrm{TM}}$ Resin (ThermoFisher). nEV concentrations were determined by Nanosight NS500 (Malvern).

\subsection{Quantification of insulin signal transduction proteins and SNP data}

Upstream insulin signal transduction proteins pS312-IRS-1, pY-IRS-1 and downstream phosphorylated (pS473-AKT, pS9-GSK3 $\beta$, pS2448-mTOR, pT389-p70S6K) and total insulin signal transduction proteins AKT, GSK3 $\beta$, mTOR, p70S6K were quantified in lysed nEVs by electrochemiluminescence in duplicate (MesoScale Diagnostics). Participant genotypes for IRS1, AKT1,GSK3B, MTOR and RPS6KB1 (encoding p70S6K) were available from a previous study [30].

\subsection{Statistics}

Statistical analyses were performed using SPSS 25.0 (IBM, Armonk, NY, USA) and G*Power (www.gpower.hhu.de). Protein measures were natural logarithm (ln)-transformed to correct skewness. Regression analysis showed significant effects of age, sex and $\ln -\mathrm{nEV}$ concentration. Group differences were assessed using a linear mixed effects model (LMM), with ln-(ECL intensity) as dependent variable, diagnosis and sex as fixed factors, age, waist-hip-ratio, cigarette smoking, and ln-nEV concentration (to normalize for differential nEV yield) as covariates, with participant ID as random effect. The primary outcome pS312-IRS-1 was analyzed first, alongside pY-IRS-1. Exploratory omnibus LMM analysis was carried out for phosphorylated and total signal transduction protein ratios (AKT, GSK3B, p70S6K) to better understand downstream cascade effects, followed by one-sided post-hoc LMM analyses for respective individual protein ratios. Next, LMM was performed with blood insulin as dependent variable and $\ln$-nEV of the respective phosphorylated to total signal transduction protein ratios as additional covariate. Pearson correlation coefficients were calculated to assess associations of protein measures with the severity of clinical symptoms (Positive and negative syndrome scale / PANSS). Due to the exploratory nature of this study we applied a significance threshold of $\mathrm{p}<0.05$ without correction for multiple comparisons. $\mathrm{P}<0.10$ was considered as trending towards significance.

To compare the genotype frequencies between DNFES and controls, SNP*diagnosis interactions were determined by F-tests. The respective $\mathrm{p}$-values were corrected for false discovery rate (FDR). 
a)

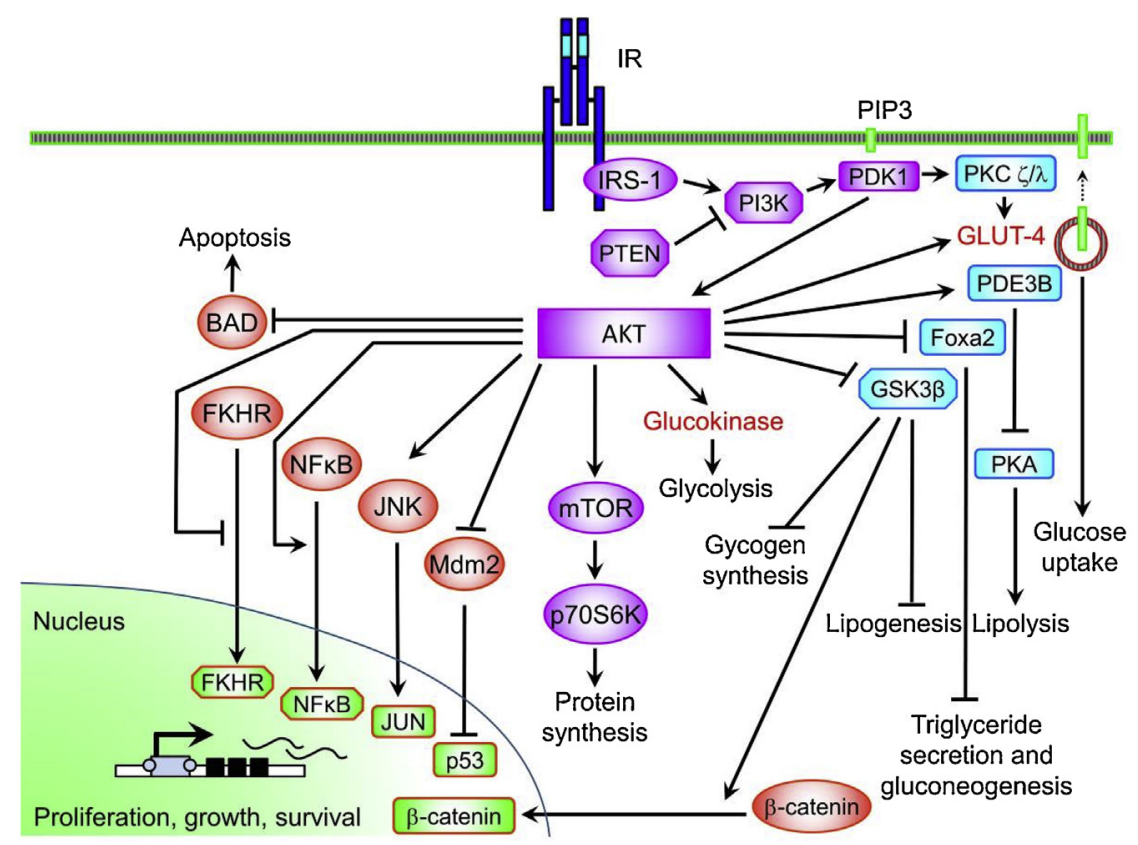

b)

Control $(n=24)$

Schizophrenia $(n=24)$

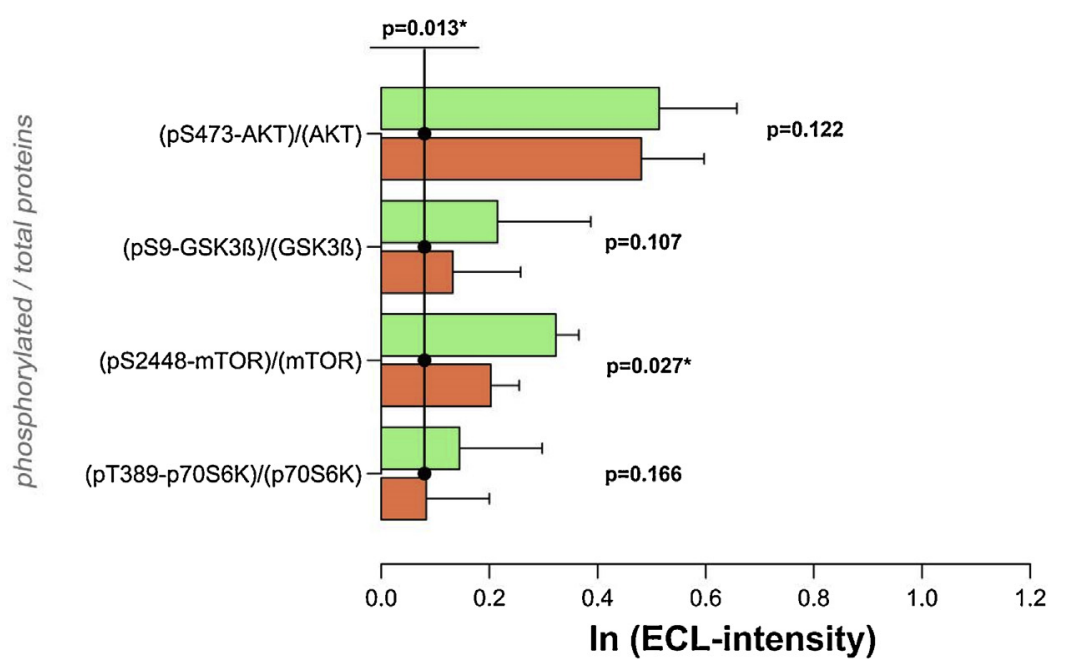

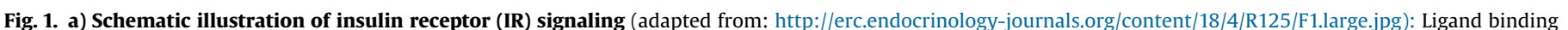

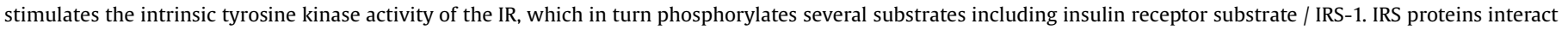

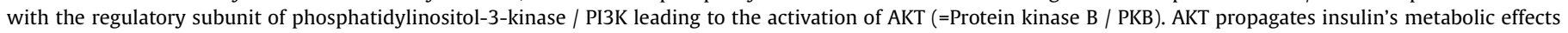

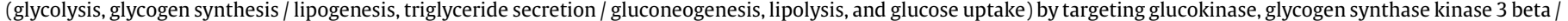

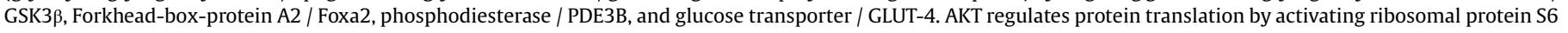

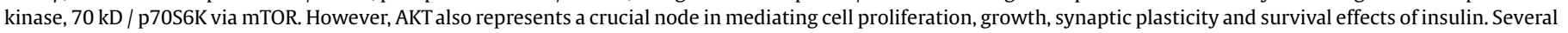

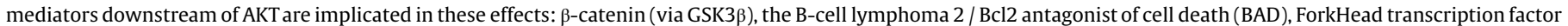

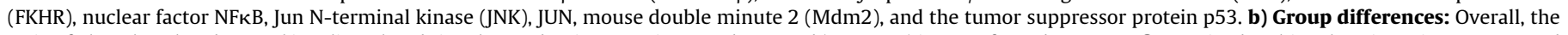

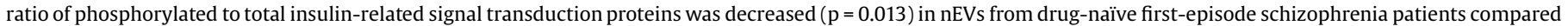

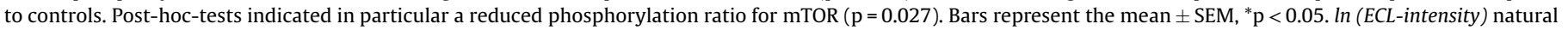
logarithm of the respective electrochemiluminescence intensity.

\section{Results}

3.1. Diagnosis-related differences regarding insulin signal transduction proteins in isolated $n E V s$

pS312-IRS-1 was lower at trend level in DNFES vs. controls $(\mathrm{p}=0.071$ ), but there was no difference for $\mathrm{pY}$-IRS. The omnibus analysis of downstream insulin signaling proteins revealed decreased phosphorylated/total signaling protein ratios in nEVs from patients vs. controls $(p=0.013)$. Post-hoc-tests indicated in particular a reduced phosphorylation ratio of $\operatorname{mTOR}(\mathrm{p}=0.027$; Fig. 1b). Regression analyses results are presented in detail with effect estimates, confidence intervals, and power analyses in Supplementary Table 2 . 
Table 1

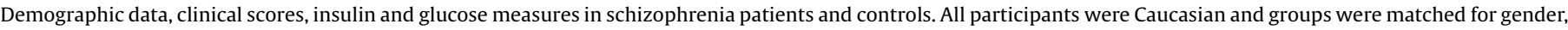
age, body mass index (BMI), and waist-hip ratio. Plasma samples were taken at $8 \mathrm{AM}$ after overnight fasting (stored at $-80^{\circ} \mathrm{C}$ ).

\begin{tabular}{|c|c|c|c|c|c|}
\hline & Controls & Schizophrenia & Test & Test value & p-value \\
\hline Sex [male / female] & $15 / 9$ & $13 / 11$ & Fisher's exact & & 0.770 \\
\hline Age [years] & $35.00 \pm 10.70$ & $32.75 \pm 11.76$ & $T$ & $\mathrm{~T}=0.693$ & 0.492 \\
\hline PANSS P score (corrected) & - & $16.21 \pm 6.57$ & & & - \\
\hline PANSS N score (corrected) & - & $11.79 \pm 9.31$ & & & - \\
\hline PANSS G score (corrected) & - & $29.00 \pm 10.62$ & & & - \\
\hline PANSS sum score (corrected) & - & $57.00 \pm 20.02$ & & & - \\
\hline Body mass index $\left[\mathrm{kg} / \mathrm{m}^{2}\right]$ & $24.31 \pm 3.33$ & $23.89 \pm 4.11$ & $T$ & $\mathrm{~T}=0.393$ & 0.696 \\
\hline Waist-hip ratio $[\mathrm{cm} / \mathrm{cm}]$ & $0.87 \pm 0.08$ & $0.86 \pm 0.06$ & $T$ & $\mathrm{~T}=0.152$ & 0.880 \\
\hline HOMA-IR [mU*mmol/L] & $0.52 \pm 0.42$ & $1.46 \pm 1.78$ & $T$ & $\mathrm{~T}=-2.513$ & 0.019 \\
\hline Insulin $[\mathbf{m U} / \mathbf{L}]$ & $2.51 \pm 2.06$ & $5.82 \pm 7.25$ & $T$ & $\mathrm{~T}=-2.151$ & 0.041 \\
\hline Glucose $[\mathrm{mmol} / \mathrm{L}]$ & $4.71 \pm 0.56$ & $5.37 \pm 1.05$ & $T$ & $\mathrm{~T}=-2.721$ & 0.010 \\
\hline Smoking [cigarettes/day] & $3.21 \pm 5.12$ & $9.37 \pm 8.13$ & $T$ & $\mathrm{~T}=-3.144$ & 0.003 \\
\hline
\end{tabular}

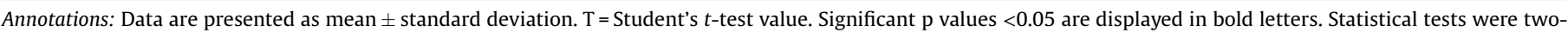

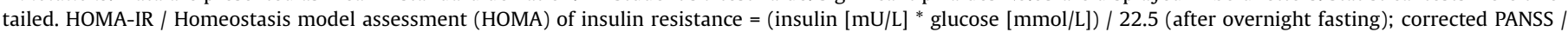

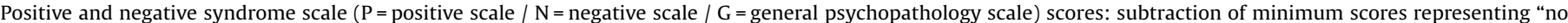
symptoms" from the PANSS scores.

\subsection{Protein phosphorylation ratios* insulin and SNP*diagnosis interactions}

The phosphorylation ratios of p70S6K $(p=0.029)$, GSK3 $\beta$ $(\mathrm{p}=0.039)$, and at trend level AKT $(\mathrm{p}=0.061)$, showed diagnosisdependent statistical interactions with insulin blood levels. No significant SNP*diagnosis interactions were identified after FDR significance adjustment (Supplementary Table 1).

\subsection{Association of insulin signal transduction protein measures with the severity of clinical symptoms}

Within the DNFES group, the ratio pS473-AKT/AKT showed a significant negative correlation with PANSS-G $(r=-0.50, p=0.016)$ and PANSS-total scores $(\mathrm{r}=-0.50, \mathrm{p}=0.015)$ and other downstream phosphorylation ratios showed similar statistical trends (correlation of PANSS-G ratios with GSK3 $\beta$ : $r=-0.038, p=0.085$; mTOR: $r=-$ $0.42, \mathrm{p}=0.055$; $\mathrm{p} 70 \mathrm{~S} 6 \mathrm{~K}: \mathrm{r}=-0.41, \mathrm{p}=0.054$; and of PANSS-total ratios with GSK3 $\beta$ : $\mathrm{r}=-0.037, \mathrm{p}=0.087$; mTOR: $\mathrm{r}=-0.24, \mathrm{p}=0.290$; p70S6K: $\mathrm{r}=-0.40, \mathrm{p}=0.058$ ).

\section{Discussion}

These findings support the hypothesis of insulin signaling abnormalities in neuronal cells in DNFES, small sample sizes notwithstanding. The observed trend towards reduced pS312-IRS1 in DNFES differs from observations in type-2 diabetes where chronic overstimulation by insulin induces an increased phosphorylation of pS312-IRS-1 via an inhibitory bottom-up feedback by elevated pT389-p70S6K [31,32]. Reduced pS312-IRS- 1 in DNFES is at first glance counterintuitive and surprising, since this condition amplifies IRS-1 signaling [31]. However, this finding may result from adaptive feedback mechanisms to primary downstream insulin signaling disturbances. In line with previous postmortem studies that point to downstream insulin signaling as the primary abnormality [1-3], the exploratory analysis of downstream signaling serine-threonine kinases (AKT, GSK3 $\beta$, mTOR, p70S6K) revealed lower phoshorylated to total protein ratios in patients vs. controls, indicating diminished signaling pathway activity; such a state could result in decreased pS312-IRS- 1 via the feedback loops of pGSK3 $\beta$ and $\mathrm{p} 70 \mathrm{S6K}$. In previous $\mathrm{nEV}$ studies examining change over time in pS312-IRS-1, AKT, GSK3 $\beta$, mTOR, and p70S6K in response to interventions, we observed similar direction of change [14,21].

Phosphorylation of serine-thereonine kinases is ATP- and pH-dependent. Thus, considering the known mitochondrial dysfunction and increased lactate production in schizophrenia [10], we hypothesize that more fundamental neuronal metabolic abnormalities underlie the observed hypophosphorylations. Phosphorylated signal transduction proteins, which are subject to dynamic regulation, were found to be reduced in DNFES, as opposed to the respective total proteins, the levels of which depend on translation (see Supplementary Figure). This discrepancy between phosphorylated and total protein levels suggests potential reversibility of neuronal insulin resistance by factors and interventions that promote insulin signaling. Accordingly, in the light of non-significant SNP*diagnosis interactions, genetic effects do not sufficiently explain the observed differences in $\mathrm{nEV}$ signaling proteins, pointing to either dominance of lifestyle/environmental factors in producing insulin signaling abnormalities in schizophrenia or the implication of unexamined and undetected variants of other genes.

Besides its brain energy metabolism role, insulin is linked to leading hypotheses of schizophrenia since 1 ) it governs brain development / maturation as well as the complexity of dendritic branching and synaptic plasticity, particularly via mTOR $[10,33]$, which showed the strongest differences among downstream signaling mediators, and 2) insulin's transduction network is shared by neurotransmitters, BNDF, and proinflammatory cytokines [34]. Thus, insulin resistance in schizophrenia may partially reflect reduced signal transduction downstream of GABA- or NMDA-receptors, dopamine-D2-recepter hyperactivity, reduced BDNF or low-grade inflammation $[10,34]$. Cerebral insulin resistance may cause partial cerebral glucose deprivation via GLUT-4 downregulation and the aforementioned shared pathways may contribute to a worsening of disease-related neurotransmitter changes [10]. Therefore, we hypothesized that the severity of psychosis symptoms might correlate inversely with the phosphorylation ratio (i.e. activity) of the tested signal transduction in our study. Indeed, changes in nEV insulin signaling biomarkers appeared to be clinically relevant, as we found associations of higher PANSS-G and PANSS-total scores with reduced phosphorylation ratios for AKT, and similar trends were observed for GSK3 $\beta$, mTOR and p70S6K. In addition, phosphorylation ratios of p70S6K, GSK3 $\beta$ and at trend level AKT showed diagnosis-dependent statistical interactions with insulin blood levels, which might contribute to our earlier observation of peripheral insulin resistance in these DNFES patients vs. controls [9].

Some limitations of our study should be noted. First, L1CAM was originally selected as target for enrichment due to its high and relatively specific expression in neural tissue and early research demonstrating high expression on EVs derived from cultured neurons [5,35]. Multifaceted evidence for neuronal and brain enrichment (i.e. increased concentration compared to control EV subpopulations for L1CAM, NCAM, synaptophysin, neurofilament-light, neuronal enolase, 
Tuj-1 and many other neuronal and some brain specific proteins) has been provided in four previous publications $[5,14,19,22]$. Nevertheless, as we previously recognized [22], L1CAM is not entirely brain-specific, since it is also highly expressed in kidney tubular epithelium [36]. Moderate L1CAM expression has been observed in peripheral nerves, intestinal crypt cells, and glandular cells of the seminal vesicle and fallopian tube (www.proteinatlas.org/ENSG00000198910-L1CAM/ tissue) and low L1CAM expression has been detected in other cell types such as lymphoid and myelomonocytic cells [37]. Therefore, the observed transduction protein differences may not be solely attributed to neurons but could also reflect disturbances of insulin signal transduction in other tissues in DNFES. Further technical optimization for specific neuronal EV enrichment is warranted to clarify this issue. Second, limited by the available sample size, the presented study was underpowered for analysis of diagnosis-related differences in individual signal transduction proteins (Supplementary Table 2) and thus we may have been unable to identify some diagnosis-related differences (false negative results). Due to the exploratory nature of our study and the relatively small sample size, correction for multiple testing for each set of analyses was not applicable, limiting the generalizability of the results. Therefore, future replication using larger cohorts is essential. The inclusion of unaffected relatives and other disorders may elucidate the etiopathological underpinnings of the present findings. However, an important strength of the study is the inclusion of well-characterized samples, with a focus on DNFES to exclude potential confounding effects of medication.

In conclusion, to our knowledge, this is the first study providing in vivo evidence for impaired insulin signaling by a comprehensive analysis of insulin signal transduction proteins in DNFES. The observed phosphorylation pattern implies that the signaling pathway activity in DNFES is compromised further downstream compared to type-2 diabetes.

\section{Author contributions}

Drs Steiner and Kapogiannis and Dipl.-Ing. Dobrowolny had full access to all study data and take responsibility for the integrity of the data and the accuracy of the data analysis ( $\mathrm{Dr}$ Kapogiannis was unblinded after $\mathrm{nEV}$ analysis was completed). Concept and design: Drs Steiner, Kapogiannis, Bernstein, Frodl, Schiltz. Acquisition, analysis, and interpretation of data: All authors. First manuscript draft: Dr Steiner. Critical revision of the manuscript for important intellectual content: All authors. Statistical analysis: Drs Kapogiannis, Steiner, Schiltz and Dipl.Ing. Dobrowolny. Administrative, technical, or material support: Drs Steiner, Kapogiannis, Mrs Tran, Mrs Mustapic. Sample collection and characterization: Mrs Meyer-Lotz and Dr. Steiner.

\section{Additional contributions}

Paul C. Guest (Cambridge, UK), PhD provided language editing of our manuscript as a native speaker. Dipl.-Psych. Josef Frank (Department of Genetic Epidemiology in Psychiatry, Central Institute of Mental Health, Mannheim, Germany) supported the analyses of participant SNP*diagnosis interactions and the search in scientific GWAS databases.

\section{Declaration of Competing Interest}

The authors declare no conflict of interest.

\section{Acknowledgments}

This research was supported in part by the Intramural Research Program of the National Institute on Aging, National Institutes of Health (Dimitrios Kapogiannis, Joyce Tran, Maja Mustapic). and by the German Federal Ministry of Education and Research (Marcella Rietschel 01EW1810).

\section{Appendix A. Supplementary data}

Supplementary material related to this article can be found, in the online version, at doi:https://doi.org/10.1016/j.eurpsy.2019.08.012.

\section{References}

[1] Chadha R, Meador-Woodruff JH. Akt-mTOR signaling pathway is downregulated in schizophrenia. Schizophr Bull 2018;44(Suppl. 1):400.

[2] Emamian ES, Hall D, Birnbaum MJ, Karayiorgou M, Gogos JA. Convergent evidence for impaired AKT1-GSK3beta signaling in schizophrenia. Nat Genet 2004:36(2):131-7.

[3] Zhao Z, Ksiezak-Reding H, Riggio S, Haroutunian V, Pasinetti GM. Insulin receptor deficits in schizophrenia and in cellular and animal models of insulin receptor dysfunction. Schizophr Res 2006;84(1):1-14.

[5] Mustapic M, Eitan E, Werner Jr. JK, Berkowitz ST, Lazaropoulos MP, Tran J, et al. Plasma extracellular vesicles enriched for neuronal origin: a potential window into brain pathologic processes. Front Neurosci 2017;11:278.

[6] Saeedi S, Israel S, Nagy C, Turecki G. The emerging role of exosomes in mental disorders. Transl Psychiatry 2019;9(1):122.

[7] Perry BI, McIntosh G, Weich S, Singh S, Rees K. The association between first episode psychosis and abnormal glycaemic control: systematic review and meta-analysis. Lancet Psychiatry 2016;3(11):1049-58.

[8] Pillinger T, Beck K, Gobjila C, Donocik JG, Jauhar S, Howes OD. Impaired glucose homeostasis in first-episode schizophrenia: a systematic review and metaanalysis. JAMA Psychiatry 2017:74(3):261-9.

[9] Steiner J, Berger M, Guest PC, Dobrowolny H, Westphal S, Schiltz K, et al. Assessment of insulin resistance among drug-naive patients with first-episode schizophrenia in the context of hormonal stress axis activation. JAMA Psychiatry 2017;74(3):968-70.

[10] Steiner J, Bernstein HG, Schiltz K, Müller UJ, Westphal S, Drexhage HA, et al. Immune system and glucose metabolism interaction in schizophrenia: a chicken-egg dilemma. Prog Neuropsychopharmacol Biol Psychiatry 2014:48:287-94.

[11] Thakore JH. Metabolic disturbance in first-episode schizophrenia. $\mathrm{Br} \mathrm{J}$ Psychiatry Suppl 2004;47:S76-9.

[12] Chouinard VA, Henderson DC, Dalla Man C, Valeri L, Gray BE, Ryan KP, et al Impaired insulin signaling in unaffected siblings and patients with firstepisode psychosis. Mol Psychiatry 2018, doi:http://dx.doi.org/10.1038/s41380018-0045-1 [Epub ahead of print].

[13] Dubal DB, Pleasure SJ. Neural-derived extracellular vesicles in clinical trials: message in a bottle. JAMA Neurol 2019;76(4):402-4.

[14] Athauda D, Gulyani S, Karnati H, Li Y, Tweedie D, Mustapic M, et al. Utility of neuronal-derived exosomes to examine molecular mechanisms that affect motor function in patients with parkinson disease: a secondary analysis of the exenatide-PD trial. JAMA Neurol 2019;76(4):420-9.

[15] Fiandaca MS, Kapogiannis D, Mapstone M, Boxer A, Eitan E, Schwartz JB, et al Identification of preclinical Alzheimer's disease by a profile of pathogenic proteins in neurally derived blood exosomes: a case-control study. Alzheimers Dement 2015;11(6):600-7.

[16] Goetzl EJ, Boxer A, Schwartz JB, Abner EL, Petersen RC, Miller BL, et al. Altered lysosomal proteins in neural-derived plasma exosomes in preclinical Alzheimer disease. Neurology 2015;85(1):40-7.

[17] Goetzl EJ, Kapogiannis D, Schwartz JB, Lobach IV, Goetzl L, Abner EL, et al Decreased synaptic proteins in neuronal exosomes of frontotemporal dementia and Alzheimer's disease. FASEB J 2016;30(12):4141-8.

[18] Kapogiannis D, Boxer A, Schwartz JB, Abner EL, Biragyn A, Masharani U, et al. Dysfunctionally phosphorylated type 1 insulin receptor substrate in neuralderived blood exosomes of preclinical Alzheimer's disease. FASEB J 2015;29 (2):589-96

[19] Kapogiannis D, Mustapic M, Shardell MD, Berkowitz ST, Diehl TC, Spangler RD, et al. Association of extracellular vesicle biomarkers with Alzheimer disease in the Baltimore longitudinal study of aging. JAMA Neurol 2019, doi:http://dx. doi.org/10.1001/jamaneurol.2019.2462 [Epub ahead of print].

[20] Mullins RJ, Mustapic M, Goetzl EJ, Kapogiannis D. Exosomal biomarkers of brain insulin resistance associated with regional atrophy in Alzheimer's disease. Hum Brain Mapp 2017;38(4):1933-40.

[21] Mustapic M, Tran J, Craft S, Kapogiannis D. Extracellular vesicle biomarkers track cognitive changes following intranasal insulin in Alzheimer's disease. J Alzheimers Dis 2019;69(2):489-98.

[22] Pulliam L, Sun B, Mustapic M, Chawla S, Kapogiannis D. Plasma neuronal exosomes serve as biomarkers of cognitive impairment in HIV infection and Alzheimer's disease. J Neurovirol 2019, doi:http://dx.doi.org/10.1007/s13365 018-0695-4 [Epub ahead of print].

[23] Wijtenburg SA, Kapogiannis D, Korenic SA, Mullins RJ, Tran J, Gaston FE, et al Brain insulin resistance and altered brain glucose are related to memory impairments in schizophrenia. Schizophr Res 2019;208:324-30.

[24] Eitan E, Tosti V, Suire CN, Cava E, Berkowitz S, Bertozzi B, et al. In a randomized trial in prostate cancer patients, dietary protein restriction modifies markers o 
leptin and insulin signaling in plasma extracellular vesicles. Aging Cell 2017:16 (6):1430-3.

[25] Pederson TM, Kramer DL, Rondinone CM. Serine/threonine phosphorylation of IRS-1 triggers its degradation: possible regulation by tyrosine phosphorylation. Diabetes 2001;50(1):24-31.

[26] Sun XJ, Goldberg JL, Qiao LY, Mitchell JJ. Insulin-induced insulin receptor substrate-1 degradation is mediated by the proteasome degradation pathway. Diabetes 1999;48(7):1359-64.

[27] Gual P, Le Marchand-Brustel Y, Tanti JF. Positive and negative regulation of insulin signaling through IRS-1 phosphorylation. Biochimie 2005;87(1):99-109.

[28] Steiner J, Fernandes BS, Guest PC, Dobrowolny H, Meyer-Lotz G, Westphal S, et al. Glucose homeostasis in major depression and schizophrenia: a comparison among drug-naïve first-episode patients. Eur Arch Psychiatry Clin Neurosci 2019;269(4):373-7.

[29] Sheehan DV, Lecrubier Y, Sheehan KH, Amorim P, Janavs J, Weiller E, et al. The Mini-International Neuropsychiatric Interview (M.I.N.I.): the development and validation of a structured diagnostic psychiatric interview for DSM-IV and ICD-10. J Clin Psychiatry 1998:59(Suppl. 20)22-33 quiz 34-57.

[30] Chan MK, Cooper JD, Heilmann-Heimbach S, Frank J, Witt SH, Nothen MM, et al. Associations between SNPs and immune-related circulating proteins in schizophrenia. Sci Rep 2017;7(1):12586.
[31] Copps KD, White MF. Regulation of insulin sensitivity by serine/threonine phosphorylation of insulin receptor substrate proteins IRS1 and IRS2. Diabetologia 2012;55(10):2565-82.

[32] Zhang J, Gao Z, Yin J, Quon MJ, Ye J. S6K directly phosphorylates IRS-1 on Ser270 to promote insulin resistance in response to TNF-(alpha) signaling through IKK2. J Biol Chem 2008;283(51):35375-82.

[33] Ryskalin L, Limanaqi F, Frati A, Busceti CL, Fornai F. mTOR-related brain dysfunctions in neuropsychiatric disorders. Int J Mol Sci 2018;19(8).

[34] Zheng W, Wang H, Zeng Z, Lin J, Little PJ, Srivastava LK, et al. The possible role of the Akt signaling pathway in schizophrenia. Brain Res 2012;1470:145-58.

[35] Faure J, Lachenal G, Court M, Hirrlinger J, Chatellard-Causse C, Blot B, et al. Exosomes are released by cultured cortical neurones. Mol Cell Neurosci 2006;31(4):642-8.

[36] Debiec H, Christensen EI, Ronco PM. The cell adhesion molecule L1 is developmentally regulated in the renal epithelium and is involved in kidney branching morphogenesis. J Cell Biol 1998;143(7):2067-79.

[37] Pancook JD, Reisfeld RA, Varki N, Vitiello A, Fox RI, Montgomery AM. Expression and regulation of the neural cell adhesion molecule L1 on human cells of myelomonocytic and lymphoid origin. J Immunol 1997;158 (9):4413-21. 